LA GRANJA:

REVISTA DE

CIENCIAS DE LA VIDA

pISSN:1390-3799; eISSN:1390-8596

http:/ / doi.org/10.17163/lgr.n27.2018.05
Artículo científico / Scientific paper

ECOLOGÍA FORESTAL

\title{
ÁRBOLES DISPERSOS Y SU EFECTO EN LA PRODUCTIVIDAD DE LOS POTREROS EN LA AMAZONÍA ECUATORIANA
}

\author{
DISPERSED TREES AND THEIR EFFECT ON THE PRODUCTIVITY OF PADDOCKS \\ IN THE ECUADORIAN AMAZONIA
}
Carlos Congo Yépez ${ }^{1 *}$, Francisco Velástegui Lara ${ }^{1}$; Carlos Caicedo Vargas ${ }^{2}$, Luis Rodríguez Iturralde ${ }^{3}$, Antonio Vera Zambrano ${ }^{4}$, Omar Montero Cruz ${ }^{1}$

\begin{abstract}
1* Programa de Ganadería y Pastos, Estación Experimental Central de la Amazonia, Instituto Nacional de Investigaciones Agropecuarias, Via San Carlos km. 1.5, sector la Parker. Telf. +593-6-3700000, La Joya de los Sachas, Orellana, Ecuador.

2 Investigador Agropecuario, Estación Experimental Central de la Amazonia, Instituto Nacional de Investigaciones Agropecuarias, Vía San Carlos km. 1.5, sector la Parker. Telf. +593-6-3700000, La Joya de los Sachas, Orellana, Ecuador. Convenio INIAP-ESPOCH-ENA.

${ }^{3}$ Programa de Ganadería y Pastos, Estación Experimental Santa Catalina, Instituto Nacional de Investigaciones Agropecuarias, Panamericana Sur km. 1, Via Tambillo, sector Cutuglagua. Telf. +593-2-3008750, Mejía, Ecuador.

${ }^{4}$ Programa de Forestería, Estación Experimental Central de la Amazonia, Instituto Nacional de Investigaciones Agropecuarias, Vía San Carlos km. 1.5, sector la Parker. Telf. +593-6-3700000, La Joya de los Sachas, Orellana, Ecuador.
\end{abstract}

*Autor para correspondencia: carlos.congo@iniap.gob.ec

\begin{abstract}
Resumen
Se seleccionaron 50 potreros con 10 años de establecimiento asociados con especies arbóreas, se realizó el inventario de las especies dispersas con diámetros de altura al pecho $(\mathrm{DAP}) \geq 5 \mathrm{~cm}$. En cada árbol se midieron las variables dasométricas DAP, altura total, área de copa. Se determinó su frecuencia, riqueza, abundancia, índice de valor de importancia, índice de biodiversidad basada en la abundancia proporcional de especies (Shannon, Simpson) y de riqueza de especies (Margalef). Se registraron 22 familias, el 83,8\% corresponden a especies maderables y el 16,2\% a especies frutales, la familia con mayor número de individuos fue Boraginaceae con 194 individuos. El DAP explico el $96 \%$ de la variación del área basal, a medida que este se incrementó y las estructuras de las especies arbóreas no demostraron influir en la producción de materia seca, cobertura y calidad de las pasturas, debido a la baja cobertura arbórea (3,69 $\pm 0,67 \%$ ) y al número de árboles dispersos en los potreros ( 5 a 7 árboles ha ${ }^{-1}$ ). El impacto de la cobertura arbórea demostró que incide significativamente en el crecimiento de las plantas, demostrando que el cultivar Marandú tiene una mejor tolerancia a la sombra respecto al Dallis y Saboya. Se concluye que los ecosistemas ganaderos Amazónicos del centro norte del Ecuador de acuerdo a los índices de biodiversidad estarían considerados como bajos, pero con una riqueza media de especies dispersas en potreros. Se identificó 4 especies con mayor peso ecológico superior al promedio (3,4\%) Cordia alliodora, Cedrela odorata, Schizolobium parahybum y Psidium guajava.

Palabras claves: Composición florística, cobertura arbórea, calidad nutritiva, índices de diversidad, índice de riqueza.
\end{abstract}




\begin{abstract}
Fifty paddocks of ten years of establishment associated with tree species were randomly selected; an inventory of all dispersed species with breast height diameters $(\mathrm{DBH}) \geq 5 \mathrm{~cm}$ was made. Dasometric variables as $\mathrm{DBH}$, total height and crown area were measured in each tree. Frecuency, richness, abundance, importance value index, biodiversity index based on proportional abundance of species (Shannon, Simpson) and species richness (Margalef) were determinated. 22 tree families were registered, $83.8 \%$ correspond to timber species and $16,2 \%$ to fruit species, the family with the highest number of individuals was Boraginaceae with $194,96 \%$ of variation of the basal area was explained by $\mathrm{BDH}$. As far as this increased and the structure of tree species do not shown any influence in dry matter production, cover and quality of the three found pasturages, due to low tree cover $(3,69 \pm 0,67 \%)$ and to the number of dispersed trees in the paddocks ( 5 to 7 trees $\mathrm{ha}^{-1}$ ). The impact of tree cover showed that it affects significantly on the growth of the plants, demonstrating that Marandú has the best shadow tolerance respect to Dallis and Saboya. It is concluded that the amazonian livestock system of northem central Ecuador according to the biodiversity indexes could be considerate as low levels, but with a medium richness of dispersed tree species on paddocks. The importance value index allowed identifying 4 species with the highest ecological weight over the average (3,4\%) Cordia alliodora, Cedrela odorata, Schizolobium parahybum and Psidium guajava.
\end{abstract}

Keywords: Floristic composition, tree cover, nutritional quality, diversity indexes, wealth indexes.

Forma sugerida de citar: Congo Yépez, C., Velástegui Lara, F., Caicedo Vargas, C., Rodríguez Iturralde, L., Vera Zambrano, A. y Montero Cruz, O. 2018. Árboles dispersos y su efecto en la productividad de los potreros en la amazonía ecuatoriana. La Granja: Revista de Ciencias de la Vida. Vol. 27(1):64-76. http://doi.org/10.17163/lgr.n27.2018.05. 


\section{Introducción}

En la Región Amazónica Ecuatoriana (RAE) las pasturas, constituyen la principal razón de cambio de uso de la tierra, desde el ecosistema original de bosque a superficie con intervención para actividades productivas, es así que el cultivo de pastizales constituye del $73 \%$ al $84 \%$ del aprovechamiento productivo del suelo en la RAE (Nieto y Caicedo, 2012).

Los sistemas tradicionales de producción ganadera en la RAE se basan en pasturas en monocultivos, que son pastoreados de manera extensiva y por lo general el componente leñoso se encuentra ausente; todo esto hace que las pasturas se lleguen a degradar y se presenten bajos indicadores productivos (MAGAP, 2014). Este tipo de sistema de producción contribuye de manera significativa una pérdida de la fertilidad natural de los suelos, contaminación de aguas, desertificación, merma de diversidad biológica y altas emisiones de gases de efecto invernadero (Tobar López y Ibrahim, 2008).

Diversos estudios demuestran que las razones más importantes para la introducción de árboles en los potreros es la provisión de sombra y alimento para el ganado, la obtención de productos como leña, madera y frutos (Esquivel et al., 2003; Restrepo, 2002). La versatilidad de los servicios que proveen los árboles y arbustos forrajeros favorecen la adopción y el diseño de sistemas silvopastoriles (SSP) (Lombo Ortíz, 2012; Esquivel et al., 2003; Restrepo, 2002).

Betancourt et al. (2003) menciona que el ganado dedica más tiempo a la rumia y al descanso en potreros con baja cobertura de árboles, lo cual influye directamente en la producción de carne y leche. Casasola Coto (2000) coincide que en sitios con mayor cobertura arbórea, los consumos del forraje se elevan hasta en un 3,7\%, además se evidencia una disminución del estrés calórico, temperatura rectal.

Estudios similares en el trópico húmedo del Ecuador han demostrado que los SSP contribuyen significativamente en la disminución de la temperatura ambiental de los potreros en horas de mayor incidencia (Criollo Rojas, 2013), aportan al mejoramiento de la calidad nutritiva y al rendimiento de forraje en base seca cuando la pastura está asociada a árboles frutales de guayaba (Psidium guajaba) como sombra y arbustos forrajeros (Leucaena leucocephala Lam.) para ramoneo (Caicedo et al., 2017; Villacis y Chiriboga, 2016).

Otros beneficios de la inclusión de árboles en pastura ya sea en forma de cercas vivas o árboles dispersos, es que pueden contribuir a la conservación de la agrobiodiversidad, funcionando principalmente como corredores biológicos para la fauna local (Beer et al., 2003).

Los árboles además contribuyen a la formación de redes estructurales, y proveen una serie de servicios ecosistémicos como fijación de carbono (Tobar López y Ibrahim, 2008). Las fincas ganaderas en la RAE con estas prácticas silvopastoriles conservan más el bosque, teniendo en promedio mayor área para la conservación (Ochoa y Valarezo, 2014).

En las provincias del centro norte de la RAE (Orellana, Napo, Sucumbíos) las fincas ganaderas con pastos naturales presentan el $43 \%$ y con pastos mejorados el $56 \%$ del área con asocio a árboles maderables y frutales (Subía et al., 2017; Virginio Filho, Caicedo y Astorga, 2014).

Con estos antecedentes el presente estudio tuvo como objetivo caracterizar el componente arbóreo (composición, riqueza, abundancia, diversidad y estructuras) en pasturas con al menos 10 años de establecimiento, además se pretende conocer el impacto de la cobertura arbórea sobre el crecimiento, cobertura, producción de fitomasa y calidad nutritiva del recurso forrajero presente en los potreros de la región centro norte de la Amazonia Ecuatoriana.

\section{Materiales y métodos}

\section{1 Área de estudio}

El estudio se realizó en fincas de pequeños y medianos productores ganaderos en áreas seleccionadas por el Proyecto "Mejoramiento de la productividad en áreas críticas de la Costa, Sierra y Amazonia", ubicadas en la Provincia de Orellana, en la parroquia La Belleza del cantón Francisco de Orellana ubicada entre los 100 y 720 msnm, a $77^{\circ} 4^{\prime} 17.84^{\prime \prime}$ y $77^{\circ} 1^{\prime} 54.27^{\prime \prime}$ de longitud oeste, $0^{\circ} 48^{\prime} 33.06^{\prime \prime}$ y $0^{\circ} 49^{\prime} 28.76^{\prime \prime}$ de latitud sur, se caracteriza por poseer suelos arcillosos de textura delgada, $\mathrm{pH}$ ácido (4.5 a 5.5) de topografía irregular, precipitación de $3126,9 \mathrm{~mm} /$ año, temperatura media anual de $26.19{ }^{\circ} \mathrm{C}$, humedad relativa del $81 \%$ GADMFO (2014); GADPRLB (2015).

En la parroquia Enokanqui del cantón La Joya de los Sachas ubicada entre 200 y $313 \mathrm{msnm}$, a $76^{\circ} 52^{\prime} 28.43^{\prime \prime}$ de longitud oeste, $0^{\circ} 12^{\prime} 52.74^{\prime \prime}$ de latitud sur, con características de suelos pardos o negros, $\mathrm{pH}$ medianamente a ligeramente ácido (5.5 a 
6.5) y una topografía plana, precipitación de 3500$4000 \mathrm{~mm} /$ año, temperatura media anual de $26.5^{\circ} \mathrm{C}$, humedad relativa del 85\% (GADMCJS, 2015; GADPRE, 2015).

\subsection{Método de investigación}

En el área de estudio se seleccionaron 50 potreros al azar con pasturas de 10 años de establecimiento asociados con especies forestales dispersas. Para la estimación del forraje producido se utilizó el promedio de 20 marcos de $1 \mathrm{~m} \times 1 \mathrm{~m}$ siguiendo transeptos en zig-zag dónde cada $10 \mathrm{~m}$ se ubicó un punto de muestreo. El análisis de materia seca y bromatología, se realizó en el laboratorio de calidad de alimentos de la Estación Experimental Central de la Amazonía (EECA).

Se realizó el inventario de todas las especies de árboles dispersas con diámetros de altura al pecho (DAP) $\geq 5 \mathrm{~cm}$ y se identificaron por especie de cada potero. En cada árbol se midieron las variables dasométricas DAP, altura total (Ht) y área de copa (Ac), mediante el cálculo perpendicular del diámetro mayor y menor. El porcentaje total de la cobertura arbórea por potrero fue estimado de la suma de todas las áreas de las copas de los árboles existentes dividida entre el área total del potrero de referencia (Villanueva Najarro et al., 2013), mediante la siguiente fórmula:

$$
C A B=\frac{\sum A c p}{A p} \times 100
$$

$\mathrm{CAB}=$ Cobertura arbórea

$\mathrm{Acp}=$ Área de copas

$\mathrm{AP}=$ Área de potrero (ha)

Se calculó el índice valor de importancia (IVI) con base a los parámetros ecológicos de abundancia relativa, dominancia relativa y frecuencia relativa, determinados de acuerdo a formulación de Stiling (1996, citado por Villavicencio Enríquez y Valdez Hernández (2003)) y Lamprecht (1962, citado por Trujillo et al. (2012)):

\section{Abundancia relativa (AR)}

$$
A R=\frac{\text { Nmero de rboles por especie }}{\text { Total de individus }} \times 100
$$

$$
F A=\frac{\text { Nmero de potreros en que aparece la especie }}{\text { Total de potreros muestreados }}
$$

Frecuencia relativa (FR)

$$
F R=\frac{\text { Frecuencia absoluta por especie }}{\text { Frecuencia de todas las especies }} \times 100
$$

\section{Dominancia (DA)}

$$
\begin{array}{r}
D A=\sum \text { Área basal }(A B) \text { de los individuos } \\
\qquad A B=\frac{\pi}{4 \times D A P^{2}}
\end{array}
$$

\section{Dominancia relativa (DR)}

$$
D R=\frac{\text { Dominancia por especie }}{\text { Dominancia de todas las especies }} \times 100
$$

Índice de valor de importancia (IVI)

$$
I V I=A R_{i}+F R_{i}+D R_{i}
$$

El análisis de diversidad se realizó mediante índices basados en la abundancia proporcional de especies (Shannon, Simpson) y de riqueza de especies (Margalef) de acuerdo a las siguientes fórmulas (Citado por Del Río et al. (2003); Trujillo et al. (2012); Martella et al. (2012):

\section{Índice de Shannon-Weaver $\left(\mathrm{H}^{\prime}\right)$}

$$
H^{\prime}=-\sum \frac{n_{i}}{N} \times \log \frac{n_{i}}{N}
$$

ni: número de individuos de la especie $\mathrm{n}$

$\mathrm{N}$ : Número total de individuos de todas las especies

\section{Índice de riqueza de Margalef ( $\mathrm{Rm})$}

$$
R m=\frac{S-1}{\log N}
$$

S: número de especies presente

$\mathrm{N}$ : número total de individuos de todas las especies

\section{Índice de dominancia de Simpson (Ds)}

$$
D s=1-\sum\left(\frac{n_{i}}{N}\right)^{2}
$$

Frecuencia absoluta (FA) 
$\mathrm{N}$ : número total de individuos de todas las especies

ni: número total de elementos pertenecientes a las especies

\subsection{Análisis estadístico}

Se empleó un análisis de varianza no paramétrico de Kruskall-Wallis para evaluar las diferencias de las variables de estructura del componente arbóreo (cobertura arbórea, área basal, densidad de árboles), se realizaron análisis de correlación de Pearson y regresión lineal entre la variable cobertura arbórea y las variables agronómicas de la pastura (altura, cobertura, materia seca, calidad nutritiva). Los análisis estadísticos se llevaron a cabo mediante el programa InfoStat (Di Rienzo et al., 2016).

\section{Resultados y discusión}

\subsection{Composición florística}

Se registraron un total de 315 individuos, pertenecientes a 29 especies y a 22 familias en un área de muestreo de 86.35 ha, se identificaron 3 pasturas asociadas a las especies arbóreas: Saboya común (Panicum maximum), representando el $37 \%$ de los potreros evaluados, seguido por el Marandú (Brachiaria Brizantha) 34\% y Dallis (Brachiaria decumbens) con el $29 \%$. El 83,8\% corresponden a especies maderables y el $16,2 \%$ a especies frutales. La familia de árboles con mayor número de especie fue Lauraceae con 3 especies (Aguacate, Canelo, Jigua), seguidas por Meliaceae (Cedro, Manzano colorado), Malvaceae (Boya, Zapote), Annonaceae (Chirimoya, Guanábana), Arecaceae (Coco, Chonta), con dos especies, mientras que la familia con mayor número de individuos fue Boraginaceae con 194 individuos, representando el $61,6 \%$, de abundancia relativa en relación al total de individuos $(\mathrm{n}=315)$, seguida por Meliaceae y Myrtaceae con 20 y 17 individuos. (Tabla 1). Subía et al. (2017) indican que los sistemas silvopastoriles más representativos que se encuentran en el centro norte de la RAE son sistemas de producción de tipo tradicional (árboles dispersos) y que en las fincas ganaderas estudiadas se encontraron especies arbóreas de tipo maderables pertenecientes a la familia Lauraceae (Laurel) y frutales de la familia Fabaceae (Guaba) representando el $43 \%$ de pasturas asociadas con sistemas silvopastoril tradicionales. Con el mismo criterio Vargas et al. (2014) menciona que los cultivos de cacao, café y ganadería con sistemas agroforestales en la RAE tienen asocio principalmente con especies forestales y frutales, lo que contribuye a la conservación de la biodiversidad y el manejo sostenible de la actividad agrícola y pecuaria de la región.

\subsection{Especies y variables dasométricas}

Tabla 1. Distribución de riqueza y abundancia de especies arbóreas en las familias registradas en potreros de la Amazonía centro norte

\begin{tabular}{lccc}
\hline \multirow{2}{*}{ Familia } & Riqueza & \multicolumn{2}{c}{ Abundancia } \\
\cline { 2 - 4 } & $\mathbf{N}^{\mathbf{o}}$. especie & Absoluta & Relativa \\
\hline Lauraceae & 3 & 13 & 4.1 \\
Meliaceae & 2 & 20 & 6.3 \\
Malvaceae & 2 & 6 & 1.9 \\
Annonaceae & 2 & 3 & 1.0 \\
Arecaceae & 2 & 10 & 3.2 \\
Myristicaceae & 1 & 2 & 0.6 \\
Moraceae & 1 & 1 & 0.3 \\
Myrtaceae & 1 & 17 & 5.4 \\
Vochysiaceae & 1 & 2 & 0.6 \\
Sapindaceae & 1 & 1 & 0.3 \\
Rutaceae & 1 & 8 & 2.5 \\
Rubiaceae & 1 & 1 & 0.3 \\
Polygonaceae & 1 & 4 & 1.3 \\
Boraginaceae & 1 & 194 & 61.6 \\
Bombacaceae & 1 & 1 & 0.3 \\
Bignoniaceae & 1 & 2 & 0.6 \\
Anacardiaceae & 1 & 3 & 1.0 \\
Caesalpinaceae & 1 & 13 & 4.1 \\
Lamiaceae & 1 & 3 & 1.0 \\
Fabaceae & 1 & 4 & 1.3 \\
Euphorbiaceae & 2 & 5 & 1.6 \\
Cecropiaceae & 1 & 2 & 0.6 \\
\hline Total & 29 & 315 & 100 \\
\hline & & & \\
\hline
\end{tabular}

En la Tabla 2 se presenta el nombre científico y común de las especies identificadas, al igual que los promedios de las mediciones dasométricas. La altura promedio fue de $7.58 \pm 0.23 \mathrm{~m}$ en un rango entre 2.15 y $28.27 \mathrm{~m}$, el $83 \%$ de las especies registraron alturas entre de 2.15 y $10.86 \mathrm{~m}$. El DAP promedio fue de $36.24 \pm 1.40 \mathrm{~cm}$ en un rango de 11.14 y $198.94 \mathrm{~cm}$, el 95\% de las especies registraron DAP entre el 11.14 y $73.74 \mathrm{~cm}$. El área de las copas registraron promedios de $73.14 \pm 5.06 \mathrm{~m}^{2}$, el $97 \%$ de las especies registraron rangos de área de copas entre el 
0.64 y $256.09 \mathrm{~m}^{2}$, la especie arbórea Higuerón (Ficus obtusifolia Kunth) fue la que registró la mayor altura $(28.27 \mathrm{~m}), \mathrm{DAP}(175.1 \mathrm{~cm})$ y área de copa $\left(604.8 \mathrm{~m}^{2}\right)$.

Los principales maderables y frutales con mayor frecuencia en los potreros de acuerdo al número de árboles (>8), registraron clases diamétricas de entre 10-38 cm (Cedro 73\%; Laurel 84\%) y 39-65 cm (Pa- chaco $84 \%$ ) para el caso de los maderable (Figura 1a). En los frutales los rangos registrados de clas0es diamétricas estuvieron comprendidos entre el 22-30 cm para el Aguacate con el 80\%; Chonta 63\%, Guayaba $47 \%$ y del $31-39 \mathrm{~cm}$ con una frecuencia para el Aguacate del 20\%; Chonta 13\%, Guayaba 29\% (Figura $1 b)$.

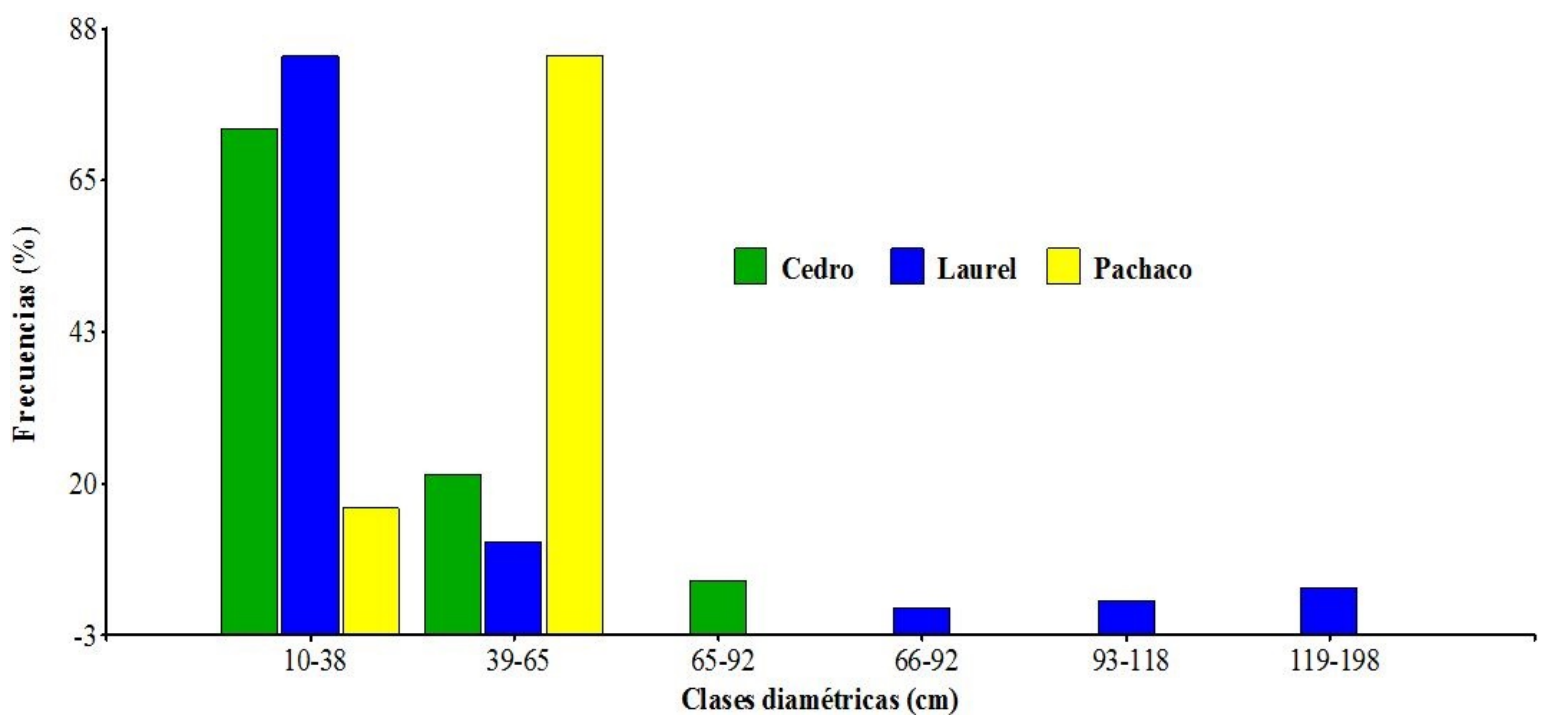

(a)

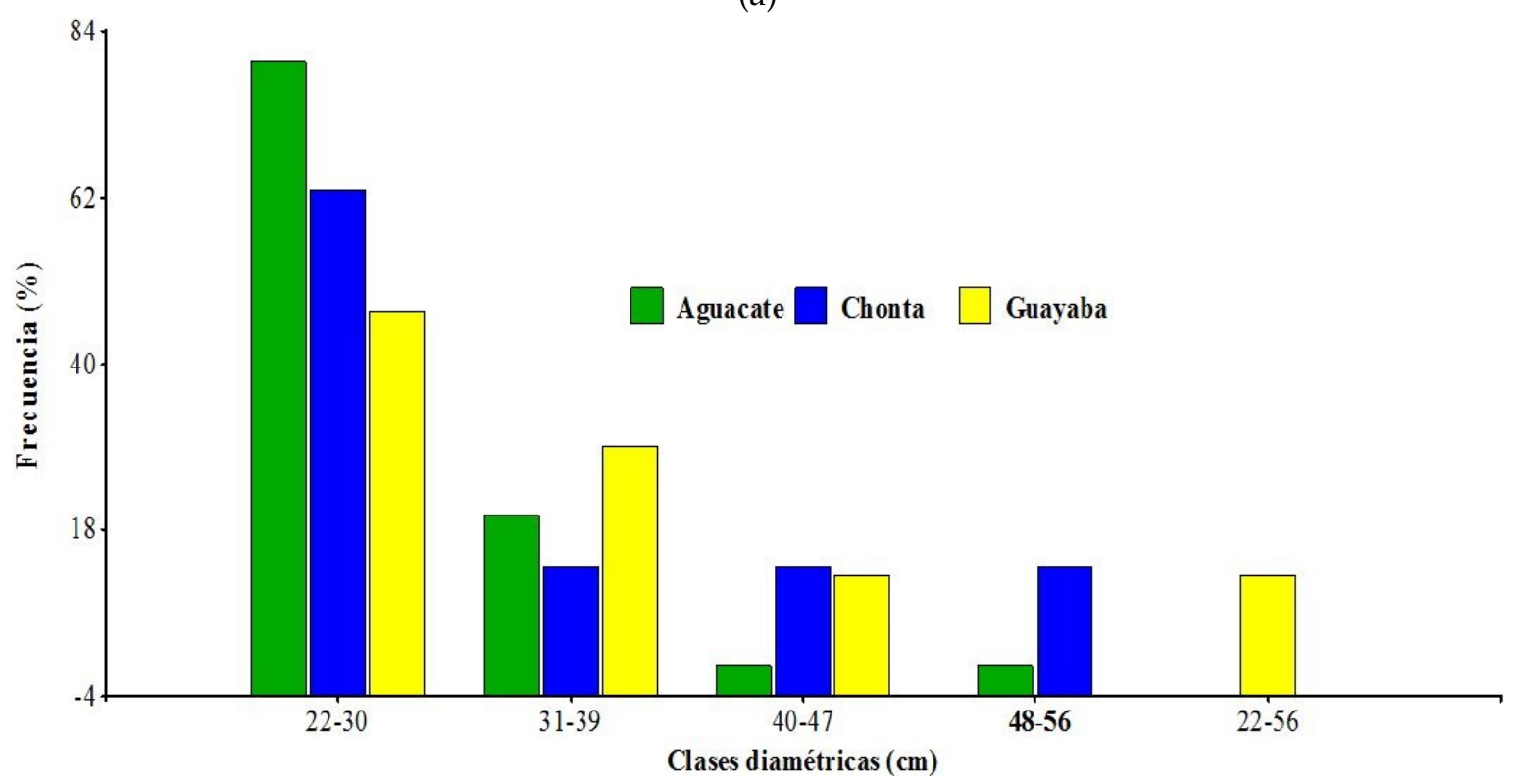

(b)

Figura 1. Clase diamétrica de los principales forestales (a) y frutales (b) en los 50 potreros evaluados del centro norte de la RAE 
Tabla 2. Especies y variables dasométricas de árboles dispersos en potreros de la Amazonía centro norte

\begin{tabular}{|c|c|c|c|c|c|}
\hline \multirow[b]{2}{*}{ Nombre científico } & \multirow[b]{2}{*}{ Nombre común } & \multicolumn{4}{|c|}{ Promedio } \\
\hline & & No. Árboles & Altura (m) & $\mathrm{DAP}(\mathrm{cm})$ & Área de copa (m2) \\
\hline Cordia alliodora & Laurel & 194 & 7.1 & 32.8 & 51.8 \\
\hline Cedrela odorata & Cedro & 19 & 7.3 & 36.9 & 52.4 \\
\hline Psidium guajava & Guayaba & 17 & 4.6 & 33.0 & 88.3 \\
\hline Schizolobium parahybum & Pachaco & 13 & 8.7 & 44.5 & 152.0 \\
\hline Bactris gasipaes & Chonta & 8 & 6.7 & 34.4 & 87.4 \\
\hline Citrus sinensis & Naranjo & 8 & 10.2 & 35.5 & 69.1 \\
\hline Nectandra hihua & Jigua & 6 & 10.9 & 45.3 & 76.9 \\
\hline Matisia cordata bonpl & Zapote & 5 & 10.8 & 46.5 & 96.8 \\
\hline Persea americana & Aguacate & 5 & 8.8 & 53.4 & 217.0 \\
\hline Myroxylon pereirae & Bálsamo & 4 & 10.2 & 37.8 & 91.8 \\
\hline Sapium sp. & Lechero & 4 & 10.6 & 44.6 & 109.9 \\
\hline Triplaris cumingiana & Fernán Sánchez & 4 & 5.2 & 33.7 & 140.3 \\
\hline Spondias anacardiaceae & Ciruelo & 3 & 8.8 & 25.3 & 37.4 \\
\hline Vitex gigantea & Pechiche & 3 & 8.7 & 32.1 & 140.1 \\
\hline Annona cherimola & Chirimoya & 2 & 8.3 & 31.0 & 58.5 \\
\hline Cecropia peltata & Guarumo & 2 & 6.8 & 34.9 & 86.8 \\
\hline Cocos nucifera & Coco & 2 & 14.8 & 70.0 & 191.8 \\
\hline Dialyanthera gracilipes & Sangre de gallina & 2 & 10.2 & 44.7 & 170.8 \\
\hline Erisma uncinatum & Arenillo & 2 & 10.8 & 82.3 & 404.4 \\
\hline Octea floribunda & Canelo & 2 & 13.9 & 66.4 & 78.2 \\
\hline Tabebuia $s p$ & Guayacán & 2 & 7.8 & 34.5 & 120.6 \\
\hline Annona muricata & Guanábana & 1 & 7.5 & 23.9 & 91.6 \\
\hline Calycophyllum spruceanum & Capirona & 1 & 8.3 & 31.2 & 208.4 \\
\hline Crescentia cujete & Lechero & 1 & 5.1 & 22.6 & 26.7 \\
\hline Ficus obtusifolia Kunth & Higuerón & 1 & 28.3 & 175.1 & 604.8 \\
\hline Guarea kunthiana & Manzano colorado & 1 & 5.4 & 27.7 & 12.9 \\
\hline Nephelium lappaceum & Achotillo & 1 & 7.2 & 95.5 & 122.7 \\
\hline Ocrhoma pyramidale & Boya & 1 & 7.9 & 82.4 & 4.0 \\
\hline Trichistandra $s p$ & Ceibo & 1 & 8.1 & 65.3 & 39.3 \\
\hline
\end{tabular}

\section{3 Índice de valor de importancia (IVI)}

El IVI permitió identificar 4 especies con mayor peso ecológico superior al promedio (34\%) y 25 especie menor al promedio. La especie forestal Laurel (Cordia alliodora) registro un IVI del 49,1\%, seguidas del Cedro (Cedrela odorata) y Pachaco (Schizolobium parahybum) con un IVI del 7,1\% y 5,5\% (Tabla 3), convirtiéndose en las 3 especies forestales con mayor importancia en los potreros de la Amazonía centro norte desde el punto de visto ecológico, abundancia, frecuencia y dominancia.

Las especies frutales ocupan el segundo orden de importancia ecológico, siendo así las especies de Guayaba (Psidium guajava), Chonta (Bactris gasipaes), Aguacate (Persea americana), Naranja (Citrus sinensis) y Zapote (Matisia cordata bonpl) con un IVI del $5,4 \% ; 3,2 \% ; 3,0 \% ; 2,8 \%$ y $2,3 \%$ respectivamente (Tabla 3). La hegemonía de estas especies está asociada a la facilidad de propagarse mediante rege- neración natural y a su alta producción de semilla. Grijalva (2012) en su informe de los recursos fitogenéticos forestales del país, menciona que el Laurel como especie nativa y el Pachaco como especie exótica están consideradas entre las 10 principales especies utilizadas con valor maderable autorizadas por el Ministerio del Ambiente (MAE), en el mismo informe hace referencia al Cedro como una de las principales especie amenazada en el país, considerando una veda de mediano plazo, entendiéndose como tal la prohibición de corte de esta especie por el MAE mediante acuerdo ministerial $\mathrm{N}^{\circ} .167$ (Presidencia de la República del Ecuador, 2007). Por otra parte Villanueva Najarro et al. (2013) menciona que especies como el Laurel, Cedro y Guayaba son mantenidas en potreros por su rusticidad ante el manejo de las pasturas, por su alta producción y fácil dispersión de semillas y por la proyección de sombra que estas especies brindan para el ganado. 
Tabla 3. Abundancia (A), dominancia (D), frecuencia (F) e índice de valor de importancia (IVI) de las especies arbóreas más comunes en potreros de la Amazonía centro norte $(\mathrm{n}=50)$

\begin{tabular}{lcccc}
\hline & \multicolumn{4}{c}{ Porcentaje (\%) } \\
\cline { 2 - 5 } Especie arbórea & $\mathrm{A}$ & $\mathrm{F}$ & $\mathrm{D}$ & $\mathrm{IVI}$ \\
\hline Cordia alliodora (Laurel) & 61.6 & 30.7 & 55.0 & 49.1 \\
Cedrela odorata (Cedro) & 6.0 & 10.0 & 5.1 & 7.1 \\
Schizolobium parahybum (Pachaco) & 4.1 & 8.0 & 4.4 & 5.5 \\
Psidium guajava (Guayaba) & 5.4 & 7.3 & 3.3 & 5.4 \\
Bactris gasipaes (Chonta) & 2.5 & 5.3 & 1.7 & 3.2 \\
Persea americana (Aguacate) & 1.6 & 3.3 & 4.0 & 3.0 \\
Citrus sinensis (Naranja) & 2.5 & 4.0 & 1.8 & 2.8 \\
Matisia cordata bonpl (Zapote) & 1.6 & 3.3 & 1.9 & 2.3 \\
Ficus obtusifolia Kunth (Higuerón) & 0.3 & 0.7 & 5.0 & 2.0 \\
Nectandra hihua (Jigua) & 1.9 & 2.0 & 2.0 & 2.0 \\
Sapium sp. (Lechero) & 1.3 & 2.7 & 1.4 & 1.8 \\
Myroxylon pereirae (Bálsamo) & 1.3 & 2.7 & 1.0 & 1.6 \\
Triplaris cumingiana (Fernán Sánchez) & 1.3 & 2.7 & 0.8 & 1.6 \\
Erisma uncinatum (Arenillo) & 0.6 & 1.3 & 2.4 & 1.4 \\
Cocos nucifera (Coco) & 0.6 & 1.3 & 1.9 & 1.3 \\
Vitex gigantea (Pechiche) & 1.0 & 2.0 & 0.6 & 1.2 \\
Octea floribunda (Canelo) & 0.6 & 1.3 & 1.5 & 1.1 \\
Spondias anacardiaceae (Ciruelo) & 1.0 & 2.0 & 0.3 & 1.1 \\
Dialyanthera gracilipes (Sangre de gallina) & 0.6 & 1.3 & 0.9 & 1.0 \\
Nephelium lappaceum (Achotillo) & 0.3 & 0.7 & 1.5 & 0.8 \\
Cecropia peltata (Guarumo) & 0.6 & 1.3 & 0.4 & 0.8 \\
Annona cherimola (Chirimoya) & 0.6 & 1.3 & 0.4 & 0.8 \\
Ocrhoma pyramidale (Boya) & 0.3 & 0.7 & 1.1 & 0.7 \\
Tabebuia sp. (Guayacán) & 0.6 & 0.7 & 0.4 & 0.6 \\
Trichistandra sp (Ceibo) & 0.3 & 0.7 & 0.7 & 0.6 \\
Calycophyllum spruceanum (Capirona) & 0.3 & 0.7 & 0.2 & 0.4 \\
Guarea kunthiana (Manzano colorado) & 0.3 & 0.7 & 0.1 & 0.4 \\
Annona muricata (Guanábana) & 0.3 & 0.7 & 0.1 & 0.4 \\
Crescentia cujete (Lechero) & 0.3 & 0.7 & 0.1 & 0.4 \\
\hline Total & 100 & 100 & 100 & 100 \\
\hline & & & & \\
\hline
\end{tabular}

\subsection{Patrones estructurales de los árboles en potreros}

La cobertura promedio de la cobertura arbórea fue de $3.69 \pm 0,67 \%$, mientras que la mayoría de los potreros $(92 \%)$ registraron coberturas arbóreas de entre 0,15 y $9 \%$ (Figura $2 b$ ) evidenciado un bajo porcentaje de sombra debido a la densidad de árboles presentes, la mayoría de los potreros ( $48 \%$ ) estuvieron comprendidos en densidades de 5 a 7 árboles $\mathrm{ha}^{-1}$ (Figura 2a), bajo este contexto se evidencia una baja presencia de árboles por unidad de superficie, Esquivel et al. (2003) ponen énfasis en que existe una gran diversidad de especies de árboles dispersos en los potreros, pero en baja densidad para no interfe- rir con la actividad ganadera, otro factor a considerar es el daño y la mortalidad de plántulas causada por el ganado en pastoreo y a la regulación de sombra por los ganaderos mediante el uso frecuente de herbicidas o chapeas (Camargo et al., 2000).

De acuerdo a Paredes y Subía (2014) en fincas diversificadas con sistemas agroforestal con cacao y café de alto potencial en la RAE, encontraron coberturas arbóreas de entre el 3 y el $76 \%$ con un promedio de $26,5 \%$, mientras que Villanueva Najarro et al. (2013) indican que en fincas ganaderas las coberturas arbóreas en potrero encontradas fueron de $10 \mathrm{y}$ $20 \%$ en el bosque subhúmedo tropical de Costa Rica. 


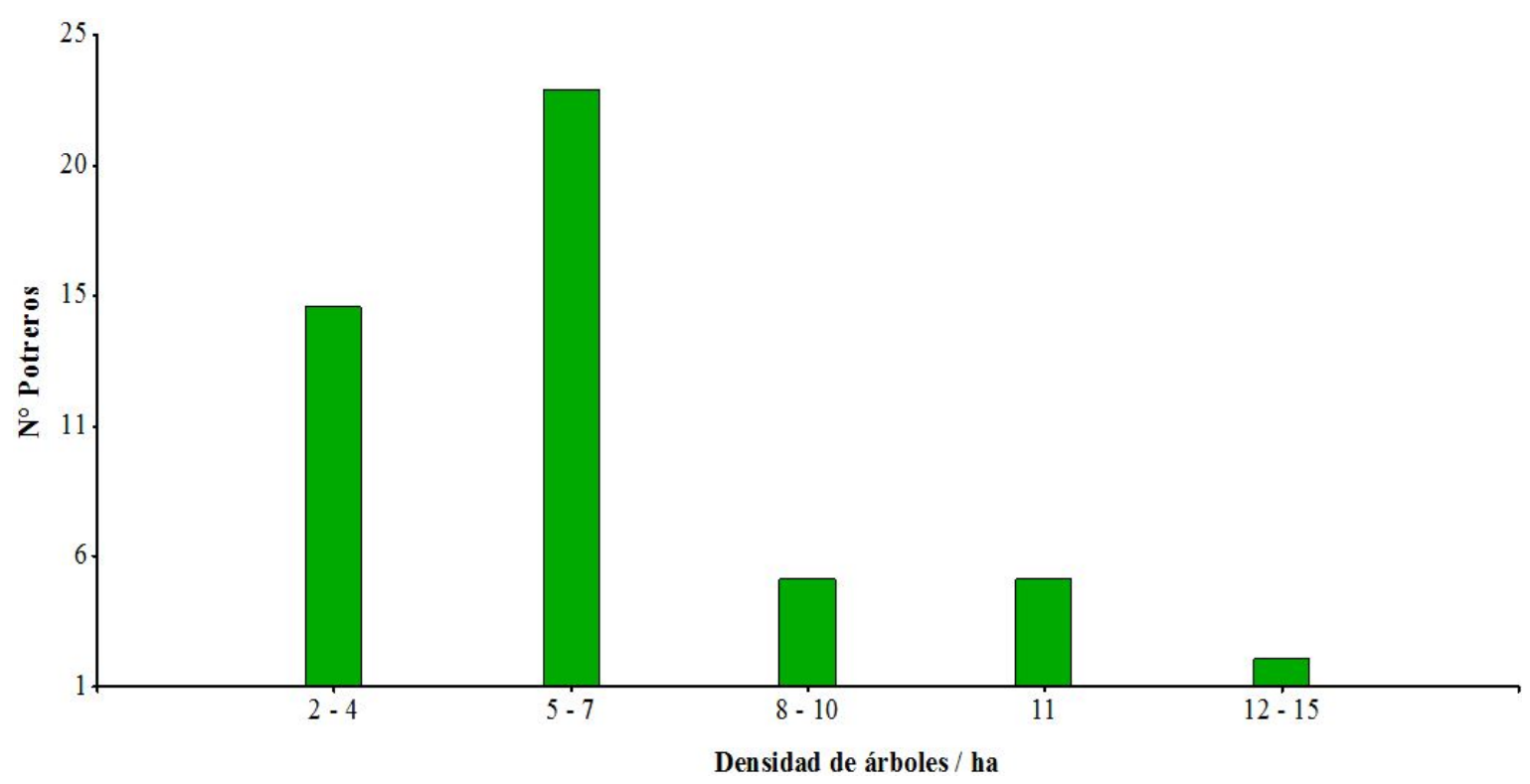

(a)

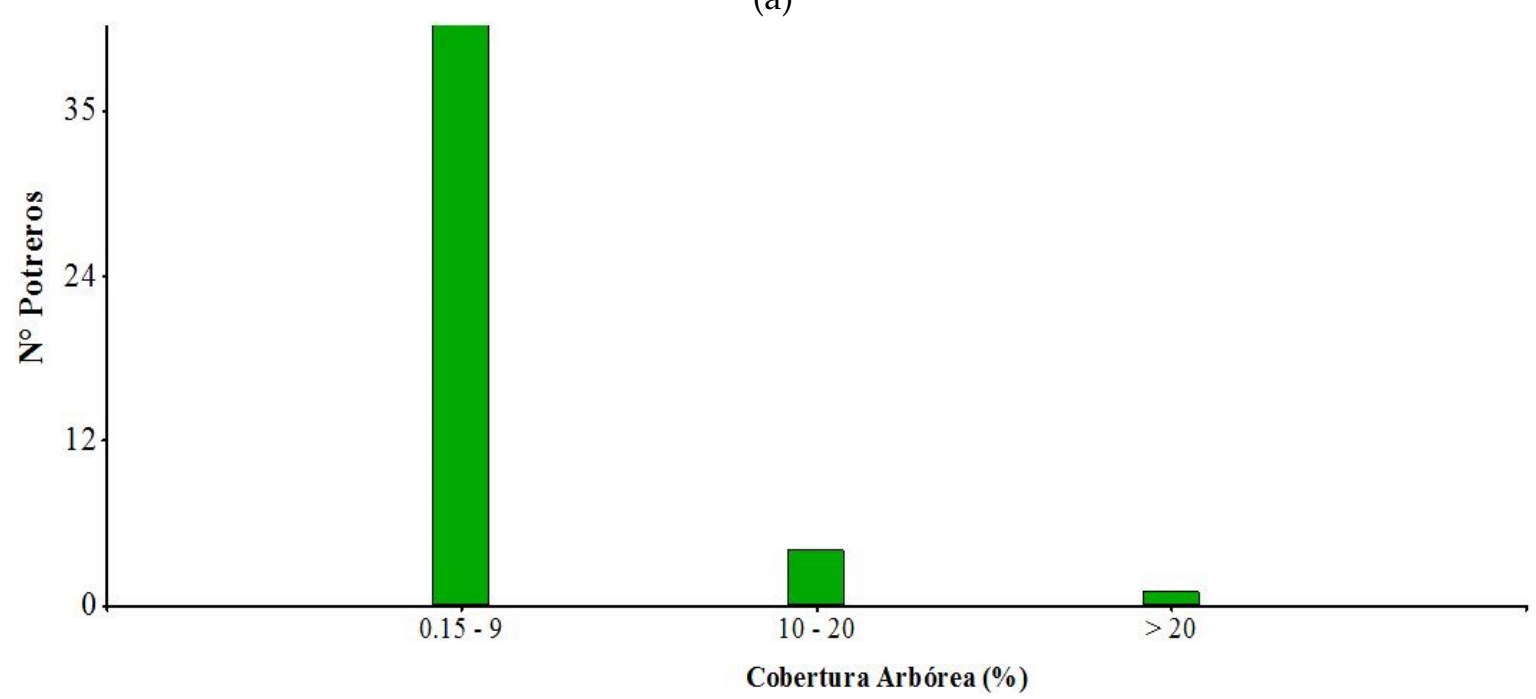

(b)

Figura 2. Distribución de la densidad (a) y la cobertura (b) de árboles/ha dispersos en los 50 potreros evaluados del centro norte de la RAE

No se encontró diferencias significativas para el área basal, cobertura arbórea, densidad de árboles ( $p>0.05$; Tabla 4), estos resultados reflejan que el manejo de la cobertura vegetal no está influenciado por el tipo de pastura. Se encontró que el DAP explicó el $96 \%$ de la variación del área basal, a medida que se incrementa (Figura 3). 
Tabla 4. Resumen de variables estructurales en tres pasturas de la Amazonía centro norte

\begin{tabular}{cccc}
\hline \multirow{2}{*}{ Variables } & \multicolumn{3}{c}{ Pastura } \\
\cline { 2 - 4 } & Dallis & Marandú & Saboya \\
Area basal $\left(\mathrm{m}^{2} \mathrm{ha}^{-1}\right)$ & $0.56 \pm 0.09 \mathrm{a}$ & $0.57 \pm 0.13 \mathrm{a}$ & $1.53 \pm 0.72 \mathrm{a}$ \\
Cobertura arbórea $(\%)$ & $4.80 \pm 1.61 \mathrm{a}$ & $3.27 \pm 0.78 \mathrm{a}$ & $3.69 \pm 1.01 \mathrm{a}$ \\
Densidad de árboles & $6.47 \pm 0.65 \mathrm{a}$ & $6.15 \pm 1.02 \mathrm{a}$ & $6.25 \pm 0.65 \mathrm{a}$ \\
\hline
\end{tabular}

Letras iguales dentro de la misma fila indican no diferencia significativa ( $p>0.05)$ según Kruskall- Wallis

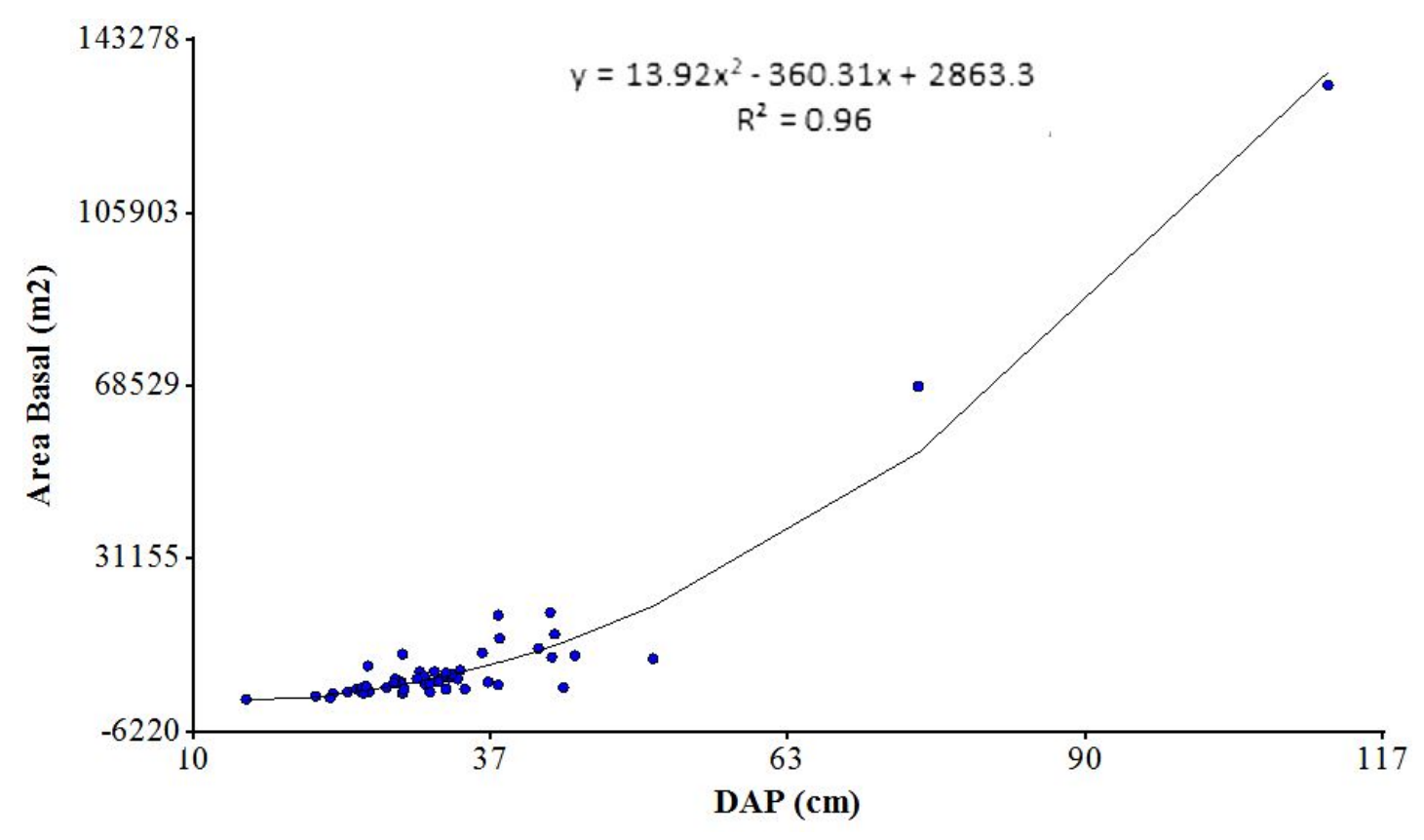

Figura 3. Incremento de DAP respecto a area basál

\section{5 Índices de diversidad estructural de las masas forestales}

Los potreros presentaron una diversidad de $\mathrm{H}^{\prime}=1.99$ de acuerdo al índice de Shannon $\left(\mathrm{H}^{\prime}\right)$, lo cual indica que los ecosistemas ganaderos de la Amazonía centro norte poseen una baja biodiversidad de árboles, resultado similar al encontrado por Paredes y Subía (2014) donde reportó valor más bajo en biodiversidad en los sistemas de producción con pasturas, debido principalmente a la poca presencia de especies forestales.

Considerando el criterio reportado por Martella et al. (2012), valores <a 2 de este indicador se consideran bajos y >a 3 son altos, los ecosistemas con mayores valores son los bosques tropicales y arreci- fes de coral, y los menores las zonas desérticas, entre mayor sea este índice el ecosistema es altamente biodiverso.

El índice de riqueza fue intermedio $(\mathrm{Rm}=3.95)$ de acuerdo al índice de Margalef ( $\mathrm{Rm})$, considerando que valores <a 2 son relacionadas a zonas con baja diversidad y valores $>\mathrm{a} 5$ zonas de alta diversidad (Campo y Duval, 2014). El índice de dominancia de Simpson (1949) (Ds) fue de 0.61 explicando la dominancia de una especie por sobre la demás, este índice está fuertemente enfocado hacia las especies más abundantes de la muestra y es menos sensible a la riqueza de especies (Martella et al., 2012; Del Río et al., 2003). 
3.6 Efecto de la cobertura arbórea sobre la productividad de la pastura

Las pasturas Dallis, Marandú, Saboya no presentaron diferencia significativas $(\mathrm{p}>0.05)$ para cobertura, materia seca y proteína cruda, al analizar el impacto del efecto de la cobertura arbórea sobre estas variables. En contraste, la altura de planta en la pastura Marandú ( $\mathrm{p}<0.05$; Tabla 5) fue significativamente mayor que Dallis y Saboya, reflejando así una mejor tolerancia a la cobertura arbórea, coincidiendo con lo reportado por Carvalho (1997), en su estudio de asociación de pasturas con árboles, concluyendo que las gramíneas que más toleraron la sombra fueron Brachiaria brizantha cv. Marandú, Panicum maximum y Brachiaria decumbens.

Tabla 5. Impacto de la cobertura arbórea sobre las variables agronómicas en tres pasturas de la Amazonía centro norte

\begin{tabular}{cccc}
\hline \multirow{2}{*}{ Variables } & \multicolumn{3}{c}{ Pastura } \\
\cline { 2 - 4 } & Dallis & Marandú & Saboya \\
\hline Altura de planta $(\mathrm{cm})$ & $78.77 \pm 1.90 \mathrm{~b}$ & $84.90 \pm 2.13 \mathrm{a}$ & $79.44 \pm 1.77 \mathrm{~b}$ \\
Cobertura $(\%)$ & $81.20 \pm 1.13 \mathrm{a}$ & $81.55 \pm 1.29 \mathrm{a}$ & $80.37 \pm 1.04 \mathrm{a}$ \\
Materia seca $\left(\mathrm{kg} / \mathrm{ha}^{-1}\right)$ & $2018.54 \pm 137.58 \mathrm{a}$ & $2187.68 \pm 157.25 \mathrm{a}$ & $2143.28 \pm 126.53 \mathrm{a}$ \\
Proteína cruda $(\%)$ & $9.77 \pm 0.50 \mathrm{a}$ & $10.16 \pm 0.57 \mathrm{a}$ & $9.34 \pm 0.46 \mathrm{a}$ \\
\hline
\end{tabular}

Letras iguales dentro de la misma fila indican no diferencia significativa $(p>0.05)$

\section{Conclusiones}

Las principales especies maderables dispersas encontrados en los potreros de la Amazonía centro norte fueron Laurel, Cedro, Pachaco y en los frutales Guayaba, Aguacate, Chonta de acuerdo a su abundancia, riqueza, abundancia y IVI. El DAP explicó el 96\% de la variación del área basal, a medida que este se incrementó y las estructuras de las especies arbóreas no demostraron influir en la producción de materia seca, cobertura, proteína cruda de las 3 pasturas encontradas, debido a la baja cobertura arbórea $(3,69 \pm 0,67 \%)$ y al número de árboles dispersos en los potreros ( 5 a 7 árboles ha ${ }^{-1}$ ), el impacto de la cobertura arbórea demostró que incide significativamente en el crecimiento de las plantas, demostrando que el cultivar Marandú tiene una mejor tolerancia a la sombra respecto al Dallis y Saboya.

Los ecosistemas ganaderos amazónicos del centro norte del Ecuador de acuerdo a los índices de biodiversidad estarían considerados como bajos, pero con una riqueza media de especies dispersas en potreros. El IVI permitió identificar las especie Laurel, Cedro, Pachaco y Guayaba con mayor peso ecológico superior al promedio $(3,4 \%)$.

\section{Agradecimientos}

Los autores expresan su agradecimiento a Junior Jiménez, Jinson Castillo, Edwin Chocho por su invaluable asistencia en el campo Al Instituto Nacional de Investigaciones Agropecuarias (INIAP) por la ejecución de esta investigación en el marco del Proyecto "Mejoramiento de la productividad en áreas críticas de la Costa, Sierra y Amazonía" financiado por la Secretaría de Educación Superior, Ciencia, Tecnología e Innovación (SENESCYT).

\section{Referencias}

Beer, J, C Harvey, M Ibrahim, J. M Harmand, E Somarriba y F. Jiménez. 2003. "Servicios ambientales de los sistemas agroforestales." Agroforestería en las Américas 10(37-38):80-87. Disponible en: https://goo.gl/rJJda4.

Betancourt, K, M Ibrahim, C Harvey y Vargas B. 2003. "Efecto de la cobertura arbórea sobre el comportamiento animal en fincas ganaderas de doble propósito en Matiguás, Matagalpa, Nicaragua." Agroforestería en las Américas 10(39-40):4751. Disponioble en: https://goo.gl/K6149R.

Caicedo, W, N Criollo, A Vera, L Riera, J Grijalva, R Ramos y C. Congo. 2017. Evaluación preliminar 
de sistemas silvopastoriles como alternativa de la producción ganadera en la Amazonía ecuatoriana. Agroforestería sostenible en la Amazonía Ecuatoriana. Disponible en: https://goo.gl/tveSMA pp. 9198.

Camargo, J. C, M Ibrahim, E Somarriba, B Finegan y D. Current. 2000. “Factores ecológicos y socioeconómicos que influyen en la regeneración natural de laurel en sistemas silvopastoriles del trópico húmedo y subhúmedo de Costa Rica." Agroforestería en las Américas 7(26):46-49. Disponible en: https://goo.gl/Rh6Luq.

Campo, A. M y V. S. Duval. 2014. “Diversidad y valor de importancia para la conservación de la vegetación natural. Parque Nacional Lihué $\mathrm{Ca}$ lel (Argentina)." Anales de Geografía de la Universidad Complutense 34(2):25-42. Disponible en: https: //goo.gl/xWtMMn.

Carvalho, M. M. 1997. "Asociaciones de pasturas con árboles en la región centro del sur de Brasil." Agroforestería en las Américas 4(15. Disponible en: https://goo.gl/UJzBne).

Casasola Coto, F. 2000. Productividad de los sistemas Silvopastoriles Tradicionales en Moropotente, Estelí, Nicaragua. Master's thesis Centro Agronómico Tropical de Investigación y Enseñanza, Costa Rica. Disponible en: https://goo. $\mathrm{gl} / 5 \mathrm{qDVPQ}$.

Criollo Rojas, N. J. 2013. Evaluación de alternativas silvopastoriles que promuevan la intensificación y recuperación de pasturas degradadas y contribuyan a reducir el impacto ambiental de la actividad ganadera en la amazonía ecuatoriana al segundo año de establecimiento. In Tesis de grado, Escuela Superior Politécnica de Chimborazo, Ecuador. Vol. Disponible en: https://goo.gl/NihpcD.

Del Río, M, F Montes, I Cañellas y G. Montero. 2003. "Revisión: Índices de diversidad estructural en masas forestales." Investigación Agraria: Sistemas y Recursos Forestales 12(1):159-176. Disponioble en: https://goo.gl/8CXsCk.

Di Rienzo, J. A, F Casanoves, M. G Balzarini, L González, M Tablada y C. W. Robledo. 2016. InfoStat versión 2016. Grupo InfoStat, FCA, Universidad Nacional de Córdoba, Argentina. Disponible en: https://goo.gl/YPxHzZ.
Esquivel, H, M Ibrahim, C. A Harvey, C Villanueva, T Benjamin y F. Sinclair. 2003. “Árboles dispersos en potreros de fincas ganaderas en un ecosistema seco de Costa Rica." Agroforestería en las Américas 10(39-40):24-29. Disponible en: https:/ / goo. $\mathrm{gl} / \mathrm{pj} 2 \mathrm{Ph} 7$.

GADMCJS. 2015. Resumen ejecutivo del Plan de Desarrollo y Ordenamiento Territorial. La Joya de los Sachas, Ecuador. In Gobierno Autónomo Descentralizado Municipal La Joya de los Sachas.

GADMFO. 2014. Plan de Desarrollo y Ordenamiento Territorial. Francisco de Orellana, Ecuador. In Gobierno Autónomo Descentralizado Municipal Francisco de Orellana.

GADPRE. 2015. Plan de Desarrollo y Ordenamiento Territorial. Enokanqui, La Joya de los Sachas, Ecuador. In Gobierno Autónomo Descentralizado Parroquial Rural Enokanqui.

GADPRLB. 2015. Plan de Desarrollo y Ordenamiento Territorial. Enokanqui, La Joya de los Sachas, Ecuador. In Gobierno Autónomo Descentralizado Parroquial Rural Enokanqui.

Grijalva, J. 2012. Situación de los Recursos Genéticos Forestales. Informe País Ecuador. Preparado por el Programa Nacional de Forestería del INIAP con aval del INIAP/FAO/MAE/MAGAP/MMRREE. Documento sometido a la Comisión Forestal de la FAO-Roma, para preparación del Primer Informe sobre el Estado de los Recursos Genéticos Forestales en el Mundo. 95 p.

Lamprecht, H. 1962. “Ensayo sobre unos métodos para el análisis estructural de los bosques tropicales." Acta Científica Venezolana 13(2):57-65. Disponible en: https://goo.gl/E3pYj2.

Lombo Ortíz, D. F. 2012. Evaluación de la disponibilidad de biomasa y capacidad de rebrote de leñosas forrajeras en potreros del trópico seco de Nicaragua. Master's thesis Centro Agronómico Tropical de Investigación y Enseñanza, Costa Rica. Disponible en: https://goo.gl/C5QSPj.

MAGAP. 2014. Proyecto agenda de transformación productiva amazónica - reconversión agroproductiva sostenible en la Amazonía Ecuatoriana. In Ministerio de Agricultura y Ganadería, Ecuador. 
Martella, M. B, E. V Trumper, L. M Bellis, D Renison, P. F Giordano, G Bazzano y R. M. Gleiser. 2012. "Manual de Ecología. Evaluación de la biodiversidad." Reduca (Biología). Serie Ecología 5(1):71115. Disponible en: https://goo.gl/VPptCq.

Nieto, C y C. Caicedo. 2012. Análisis reflexivo sobre el desarrollo agropecuario sostenible en la amazonía ecuatoriana. In Ecuador: INIAP, Estación Experimental Central de la Amazonia. (Publicación Miscelánea no. 405). Disponible en: https://goo.gl/co89yJ.

Ochoa, D y J. Valarezo. 2014. “Caracterización y análisis de rentabilidad de los sistemas de producción ganaderos presentes en el cantón Yantzaza, Ecuador." CEDAMAZ 4(1):76-85. Disponible en: https://goo.gl/enVhwi.

Paredes, N y C Subía. 2014. Valoración de los servicios ambientales en fincas diversificadas con sistemas agroforestales de alto potencial. Agroforestería sostenible en la Amazonía Ecuatoriana. Disponible en: https://goo.gl/tveSMA pp. 73-90.

Presidencia de la República del Ecuador. 2007. Registro oficial $\mathrm{N}^{\circ} 18$ - jueves 08 de febrero del 2007. In Dado en el Palacio Nacional al 25 de noviembre del 2009. Quito, EC.

Restrepo, S. 2002. Relaciones entre la cobertura arbórea en potreros y la producción bovina en fincas ganaderas en trópico seco en Cañas, Costa Rica. Master's thesis Centro Agronómico Tropical de Investigación y Enseñanza, Costa Rica. Disponible en: https:/ / goo.gl/SPqrqG.

Simpson, E. H. 1949. "Measurement of Diversity." Nature, International journal of science 163, 688.: doi: https://www.dx.doi.org/10.1038/ $163688 \mathrm{a} 0$.

Stiling, P. D. 1996. Ecology; Theories and Applications. Prentice Hall series in the life sciences. Disponible en: https://goo.gl/14Efdm.

Subía, C, N Paredes, W Caicedo, F Fernández, A Díaz, F Bastidas y J. Chávez. 2017. Análisis socioproductivo de los sistemas de producción agropecuaria en la zona norte y centro de la Amazonía Ecuatoriana. Agroforestería sostenible en la Amazonía Ecuatoriana. Disponible en: https://goo.gl/tveSMA pp. 49-58.
Tobar López, D y M. Ibrahim. 2008. "Valor de los sistemas silvopastoriles para conservar la biodiversidad en fincas y paisajes ganaderos en América Central." Centro Agronómico Tropical de Investigación y Enseñanza (CATIE), Costa Rica. Disponible en: https://goo.gl/326cRe.

Trujillo, L. A, Y. K Cuellar, D. Y Huaca, J. E Velásquez y J. C. Suarez. 2012. "Caracterización de árboles dispersos en potreros y su efecto en la cobertura herbácea en pasturas del piedemonte amazónico colombiano." Revista Momentos de Ciencia 9(1):50-58. Disponible en: https:/ / goo.gl/ u6sqSQ.

Vargas, Y, P Jaramillo, Y Sánchez y D. Sotomayor. 2014. Valoración socio-productiva de fincas diversificadas con sistemas agroforestales de alto potencial. Agroforestería sostenible en la Amazonía Ecuatoriana. Disponible en: https://goo.gl/tveSMA pp. 59-72.

Villacis, J y C. Chiriboga. 2016. “Relaciones entre las variables socioeconómicas y la cobertura arbórea de fincas ganaderas del trópico húmedo del Ecuador." Revista Cubana de Ciencias Forestales 4(2):149-163. Disponible en: https://goo.gl/ 8DMqtH.

Villanueva Najarro, C, D Tobar López, M Ibrahim, F Casasola Coto, J Barrantes y R. Arguedas. 2013. "Árboles dispersos en potreros en fincas ganaderas del Pacífico Central de Costa Rica." Agroforestería en las Américas (45):12-20. Disponible en: https://goo.gl/J9Kw5R.

Villavicencio Enríquez, L y J. Valdez Hernández. 2003. "Análisis de la estructura arbórea del sistema agroforestal rusticano de café en san miguel, Veracruz, México." Agrociencia 37(4):413-423. Disponible en: https://goo.gl/eFojC7.

Virginio Filho, E, C Caicedo y C. Astorga. 2014. Agroforestería sostenible en la Amazonía ecuatoriana. Serie técnica N 398. Centro Agronómico Tropical de Investigación y Enseñanza (CATIE) y Instituto Nacional Autónomo de Investigaciones Agropecuarias (INIAP). Disponible en: https://goo.gl/ tveSMA. 